\title{
The Research of Passenger Flow Through Checkpoint Based on Queuing Theory
}

\author{
Jie Yi \\ North China Electrical Power University
}

Keywords: Queuing theory; modeling methods; passenger flow

\begin{abstract}
Long lines emerging recently at American airports incurred sharp criticism for the TSA, how to solve the problem becomes a concern both for the TSA and passengers. To explore the flow of passengers through a security checkpoint, propose some measures to modify the current process and some advice for managers,we develop modeling methods based on queuing theory. Queue length l, queuing probability $\mathrm{c}$ and average waiting time $\mathrm{w}$ can be obtained by solving equations. We then explore the relations between these variables and the passenger flow $\lambda$ and give an expression $\lambda=w \cdot c / l$.
\end{abstract}

\section{Introduction}

Recently, lines in America seems to be insane,which frustrates many travelers.A recent survey conducted among American on the Internet says that compared with queuing at airports, 35\% of participants prefer to do shopping, 22\% prefer to clean up the mess after holiday picnics,and $18 \%$ would like to sleep in the sofa ${ }^{[1]}$.So it is not difficult to see passengers' anxiety considering hours-long security wait time. This algorithm is also a part of our model regarding the passenger flow through the checkpoint.We set up parameters $\lambda$ : passenger arrival rate, $u$ :service rate,and utilization rate of the service desk $\rho$ as criteria.

\section{Research results based on Queuing Theory:}

Poisson process is a special random process which is frequently used to describe customer arrival disciplines in queuing theory. The probability of customers arriving in from $t_{1}$ to $t_{2}$ can be described by following formula,on the premise that the number of customers is $n$ :

$P n\left(t_{1}, t_{2}\right)=P\left\{N\left(t_{2}\right)-N\left(t_{1}\right)=n\right\} \quad\left(t_{1}<t_{2} ; n \geq 0\right)$

where

$N(t)$ is the number of customers arriving in from 0 to $t$

$\operatorname{Pn}\left(t_{1}, t_{2}\right)$ follows the Poisson distribution with the parameter $\lambda_{1}$ when it meets one of the three following conditions:(1)stability(2)independence(3)universality ${ }^{[2]}$.

Through a series of deduction,the probability of $n$ customers arriving in during the $t$ interval $P_{n}(t)$ is described as:

$$
P_{n}(t)=\frac{\left(\lambda_{1} t\right)^{n}}{n !} e^{-\lambda_{1} t} \quad(t>0 ; n=0,1,2 \cdots)
$$

so we can see $N(t)$ follows the Poisson distribution,its mathematical expectations and variances are as follows:

$$
E(N(t))=\sum_{n=0}^{\infty} n \frac{\left(\lambda_{1} t\right)^{n}}{n !} e^{-\lambda_{1} t}=\lambda_{1} t, \operatorname{Var}(N(t))=\lambda_{1} t
$$

especially, when $t=1$, we can get $E(N(1))=\lambda_{1}$, which represents average number of arrivals per unit time, also called the arrival rate ${ }^{[3]}$.

If the probability density function of random variable $T$ is expressed as 


$$
f(t)=\left\{\begin{array}{ll}
\mu e^{-\mu t} & (t \geq 0) \\
0 & (t<0)
\end{array} \quad(\mu>0)\right.
$$

then $T$ follows the negative exponential distribution with parameter $\mu$.

The distribution function is described as

$$
F(t)=\left\{\begin{array}{ll}
1-\mu e^{-\mu t} & (t \geq 0) \\
0 & (t<0)
\end{array}(\mu>0)\right.
$$

next

$$
\begin{aligned}
& E(T)=\frac{1}{\mu} \\
& \operatorname{Var}(T)=\frac{1}{\mu^{2}}
\end{aligned}
$$

are easy to obtain. Where $\mu$ stands customers who have received service and leave the system per unit time,also called the average service rate of each check-in desk.

Assuming that each check-in desk work independently and average service rate is equal,namely $\mu_{1}=\mu_{2}=\cdots=\mu_{c}=\mu$.So average service rate of the whole service agent is either $c \mu$ when $n \geq c$ or $n \mu$ when $n \prec c$. We define average utilization of service agent as

$$
\rho=\frac{\lambda_{1}}{c \mu}
$$

to prevent an infinite queue, $\rho$ needs to satisfy $\rho<1$.Fig.1 shows the process.

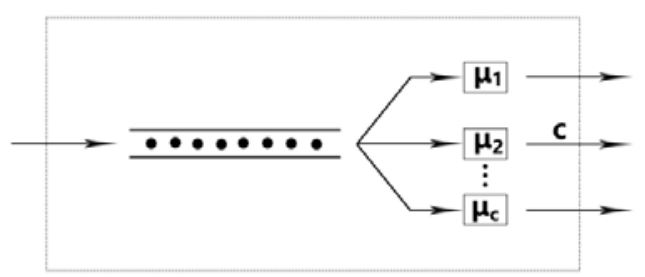

Fig.1 a sketch of service desks

Begin analysis of the queuing system from investigating the relation of system states.The transfer relations between theses states are shown as figure1.

The transition from state 0 to state 1 means that a customer has received service and leaved,the state transition probability is represented by $\mu P_{1}$. The transition from state 2 to state 1 means that one of customers served at the service desk $\mu_{1}$ and $\mu_{2}$, and the state transition probability is $2 \mu P_{2}$.

Similarly,consider the case where the state transfer from state $n$ to state $n-1$. The state transition probability is $n \mu P_{n}$ in the case of $n \leq c$. While if $n>c$, limited number of service desks determine that there are $c$ customers being at service at most,and $n-c$ customers waiting,the state transition probability is therefore changed into $c \mu P_{n}$.

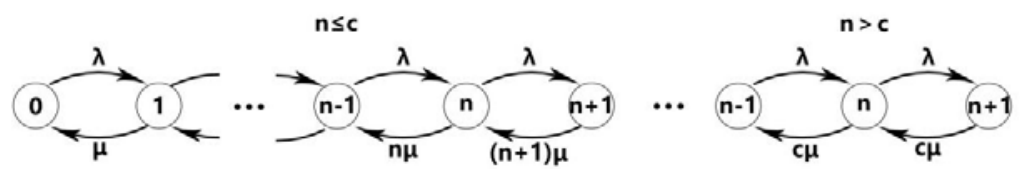

Fig.2 state transition process

From figure2 we can conclude that

$$
\begin{cases}\mu P_{1}=\lambda_{1} P_{0} & \\ (n+1) \mu P_{n+1}+\lambda_{1} P_{n-1}=\left(\lambda_{1}+n \mu\right) P_{n} & (1 \leq n \leq c) \\ c \mu P_{n+1}+\lambda_{1} P_{n-1}=\left(\lambda_{1}+c \mu\right) P_{n} & (n>c)\end{cases}
$$


Solving the deterministic difference équations using recursion gives state probability as follows

$$
\begin{gathered}
P 0=\left[\sum_{k=0}^{c-1} \frac{1}{k !}\left(\frac{\lambda_{1}}{\mu}\right)^{k}+\frac{1}{c !} \frac{1}{1-\rho}\left(\frac{\lambda_{1}}{\mu}\right)^{c}\right]^{-1} \\
P n= \begin{cases}\frac{1}{n !}\left(\frac{\lambda_{1}}{\mu}\right)^{n} P_{0} & (n \leq c) \\
\frac{1}{c ! c^{n-c}}\left(\frac{\lambda_{1}}{\mu}\right)^{n} P_{0} & (n>c)\end{cases}
\end{gathered}
$$

system running indexes are derived as:

average queue length

$$
\left\{\begin{array}{l}
L_{s}=L_{q}+\frac{\lambda_{1}}{\mu} \\
L_{q}=\sum_{n=c+1}^{\infty}(n-c) P_{n}=\frac{(c \rho)^{c} \rho}{c !(1-\rho)^{2}} P_{0}
\end{array}\right.
$$

average waiting time and sojourn time derived from the Little formula are shown as follows respectively

$$
W_{q}=\frac{L_{q}}{\lambda_{1}}, \quad W_{s}=\frac{L_{s}}{\lambda_{1}}
$$

The results are given in terms of requirements and reality,intermediate data processing are omitted for simplicity,the results are given as follows

average queue length

$$
\left\{\begin{array}{l}
L_{q}=\frac{\rho^{k+1}}{(k-\rho) \sum_{n=0}^{k-1} C_{n} \rho^{n}} \\
C_{n}=(k-1) ! \frac{n-k}{n !}
\end{array}\right.
$$

average waiting time

$$
W_{q}=\frac{L_{q}}{\lambda_{1}}
$$

queuing probability

$$
C(\rho, k)=\frac{k-\rho}{\rho} L_{q}
$$

\section{References}

[1]. Tosic V. A review of airport passenger terminal operations analysis and modelling[J]. Transportation Research Part A: Policy and Practice, 1992, 26(1): 3-26.

[2]. Schelasin, Roland E A. Capacity Management Using Static Modeling, Queuing Theory, and Performance Curves (Presentation)[J]. IIE Annual Conference. Proceedings,2013,

[3]. H.L WANG. Model of Gun-missile Anti-tank Operational Efficiency based on Bidirectional Queuing Theory[A]. International Research Association of Information and Computer Science.Proceedings of International Conference on Industrial Technology and Management Science(ITMS 2015)[C].International Research Association of Information and Computer Science:,2015:4. 